\title{
Value of CT-Based Texture Analysis in Preoperative Prediction of the Grade of Gastrointestinal Stromal Tumors Compared to Conventional CT Imaging
}

\author{
Caiyue Ren ${ }^{1}$, Shengping Wang ${ }^{1}$, Shengjian Zhang ${ }^{1,{ }^{*}}$ and Zhaoxia Jiang ${ }^{1}$ \\ ${ }^{1}$ Shanghai Cancer Center, Fudan University, Shanghai, China \\ "Corresponding author: Shanghai Cancer Center, Fudan University, Shanghai, China. Email: shengjianz@163.com
}

Received 2018 October 24; Revised 2019 August 26; Accepted 2019 September 04.

\begin{abstract}
Background: Gastrointestinal stromal tumors (GISTs), which are the most common mesenchymal tumors of the digestive system, are classified as very low, low, intermediate and high risk. The treatment and prognosis of GISTs vary according to the grade. Objectives: To investigate the capability of computed tomography (CT)-based texture analysis for predicting the grade of GISTs, and compare the findings with a combination model consisting of CT signs and texture parameters.

Patients and Methods: For this retrospective study, a total of 168 patients (training group: $n=117$; validation group: $n=51$ ) with pathologically proven GISTs were analyzed. Patients were randomly divided into the potential malignant and malignant group. Radiomics signature based on texture features and the combination model consisting of selected CT signs and texture parameters were developed with the least absolute shrinkage and selection operator (Lasso) regression. The prediction performance of models was evaluated by the area under the receiver operating characteristic (ROC) curve(AUC). Calibration was evaluated with the HosmerLemeshow goodness-of-fit test.

Results: Totally, 29 texture features and seven CT signs were extracted. Texture features of sphericity, compacity, contrast, and dissimilarity, and CT signs of size, and location were selected to develop the predictive models. The rad-and pre-scores were calculated for the radiomics signature and combination model based on the validation group. Both two models hold great prediction performance with AUCs of 0.897 and 0.959 ( $\mathrm{P}<0.05$ ), sensitivities of $76.20 \%$ and $90.50 \%$, specificities of $90.0 \%$ and $93.30 \%$, accuracies of $84.30 \%$ and $90.20 \%$, respectively. The combination model performed better. Calibration curves showed no statistically significant differences between the two models $(\mathrm{P}>0.05)$.

Conclusion: The prediction models were validated to be valuable for risk grade of GISTs and may provide non-invasive and practical biomarkers for optimizing the treatment strategy and improving the prognosis. In addition, the combination model had more advantages than texture analysis alone.
\end{abstract}

Keywords: Gastrointestinal Stromal Tumor, Risk, Computed Tomography, X-Ray, Texture Analysis

\section{Background}

Gastrointestinal stromal tumors (GISTs), which are the most common mesenchymal tumors of the digestive system with malignant potential (1), are classified as very low, low, intermediate and high risk according to the $2008 \mathrm{Na}$ tional Institutes of Health (NIH) criteria (2). Asian Consensus Guidelines suggest that very low- and low-risk GISTs should be treated as benign tumors under strict monitoring, while patients with intermediate- and high-risk GISTs are required to take imatinib mesylate in addition to the operation to prevent metastasis or postoperative recurrence (3). Needle biopsy for immunological analysis is commonly used in clinical practice. However, improper operation might cause tumor rupture and hemorrhage, increasing the risk of tumor dissemination (4). Thus, it is clinically important and necessary to explore noninvasive, reliable and practical markers for preoperatively predicting the grade in patients with GISTs.

Currently, at least three imaging methods are used for GIST patients. Although positron emission tomographycomputed tomography (PET/CT) is the most sensitive and accurate method, it is not recommended as a routine examination because of the high cost (5). Magnetic resonance imaging (MRI) may be another method that could provide functional quantitative indicators but its clinical significance in this context requires further study (6). 
Computed tomography (CT) is widely recognized as the main imaging method due to its moderate price and convenient operation. CT images can show the location and size of masses, as well as the presence of cystic necrosis and distant metastases (7). However, these signs are assessed based on experience, which are greatly influenced by subjective factors, and cannot directly predict the grade in patients with GISTs.

Texture analysis as a quantitative imaging technique could better avoid diagnostic errors caused by human factors compared to conventional CT examination (8), and it could objectively reflect the potential biological characteristics and heterogeneity of tumors (9). Texture analysis based on CT, MRI and PET/CT scan has achieved preliminary results for grade in the head and neck, and rectal cancer $(10,11)$. Recent reports have shown that texture analysis was of certain value in the diagnosis and differentiation of gastric tumors $(12,13)$.

\section{Objectives}

The purpose of this study was to investigate the capability of CT-based texture analysis for predicting the grade of GISTs and compare the findings with those of a combination model consisting of CT signs and texture parameters.

\section{Patients and Methods}

\subsection{Patients}

The medical ethics committee of our institution approved this study. For this retrospective study, the requirement for informed consent was waived. A total of 873 patients with pathologically confirmed GISTs from January 2011 to September 2017 in Fudan University Shanghai Cancer Center, Shanghai, China were selected for this study. The patient recruitment process is presented in Figure 1. Finally, 168 patients ( 88 females and 80 males) were included in the final analysis. The median age was 57 years with a range of 22 to 93 years. Patients were divided into training group ( $n=117)$ and validation group $(n=51)$ after simple randomization at a ratio of 7 to 3.

\subsection{Pathological Characteristics}

All tumors were evaluated for histological characteristics and the expression of CD117 and CD34. The tumors were divided into the potential malignancy group (very low and low risk) and the malignancy group (intermediate and high risk).

\subsection{CT Image Acquisition and Analysis}

Patients generally underwent contrast-enhanced CT scans less than 10 days before treatment on the 32- or 64slice Siemens Sensation system, and the scan range included all lesion areas. The CT parameters were as follows and used with a standard reconstruction algorithm: tube voltage, $120 \mathrm{kVp}$; tube current, 250 - $300 \mathrm{~mA}$; slice thickness and interval, $1.0 \mathrm{~mm}$. A total of $80-120 \mathrm{~mL}(1.5 \mathrm{~mL} / \mathrm{kg})$ of iodinated contrast material was injected at a flow rate of $3 \mathrm{~mL} / \mathrm{s}$ into the antecubital vein. Considering that the masses in this study were distributed in different segments of the digestive tract, and were observed more clearly in the portal venous phase, the texture features extraction were only performed on portal venous phase CT images in this study. There was no universally accepted criterion for the selection of scanning phase for texture analysis, but majority of the reports nevertheless selected portal venous phase CT images $(12,14,15)$. The portal venous phase CT images were performed at $60 \mathrm{~s}$ after injection of contrast medium.

Two radiologists with 3 and 13 years of experience in CT diagnosis assessed the following image signs of each mass without knowing the pathology determined by consensus: tumor location (gastric and nongastric), size (the maximal diameter of the largest cross section $<5 \mathrm{~cm}, 5-10 \mathrm{~cm}$ or $>10 \mathrm{~cm}$ ), growth pattern (inter-intestinal, extra-intestinal or cross-intestinal), shape (regular or irregular), boundary (clear or unclear), calcification and cystic necrosis (present or absent) (16).

\subsection{Texture Feature Extraction and Analysis}

Feature extraction was performed using the LIFEx (LIFEx3.40, CEA-SHFJ, Orsay, France) package. The above two radiologists selected the largest slice of the tumor to delineate the region of interest (ROI) on the transverse section by consensus (Figure 2), and the coronal and sagittal positions of the ROI were obtained automatically. The ROI selection included all tumors and avoided blood vessels, calcification and gas. A total of 29 features were extracted automatically including: (a) two shape parameters, (b) four histogram parameters, (c) four conventional parameters, (d) six gray-level co-occurrence matrix (GLCM) parameters, (e) 11 grey level run length matrix (GLRLM) parameters, (f) two neighborhood gray-level different matrix (NGLDM) parameters. A list of the corresponding features is provided in Table 1, while a detailed description of all features have been mentioned in a study by Orlhac et al. (17). 
Inclusion criteria:

1) patients who underwent surgery for

GISTs with curative intent;

2) information of postoperative

pathologically comfirmed GISTs risk

grading available;

than 10 days before treatment.
3) standard contrast-enhanced CT less

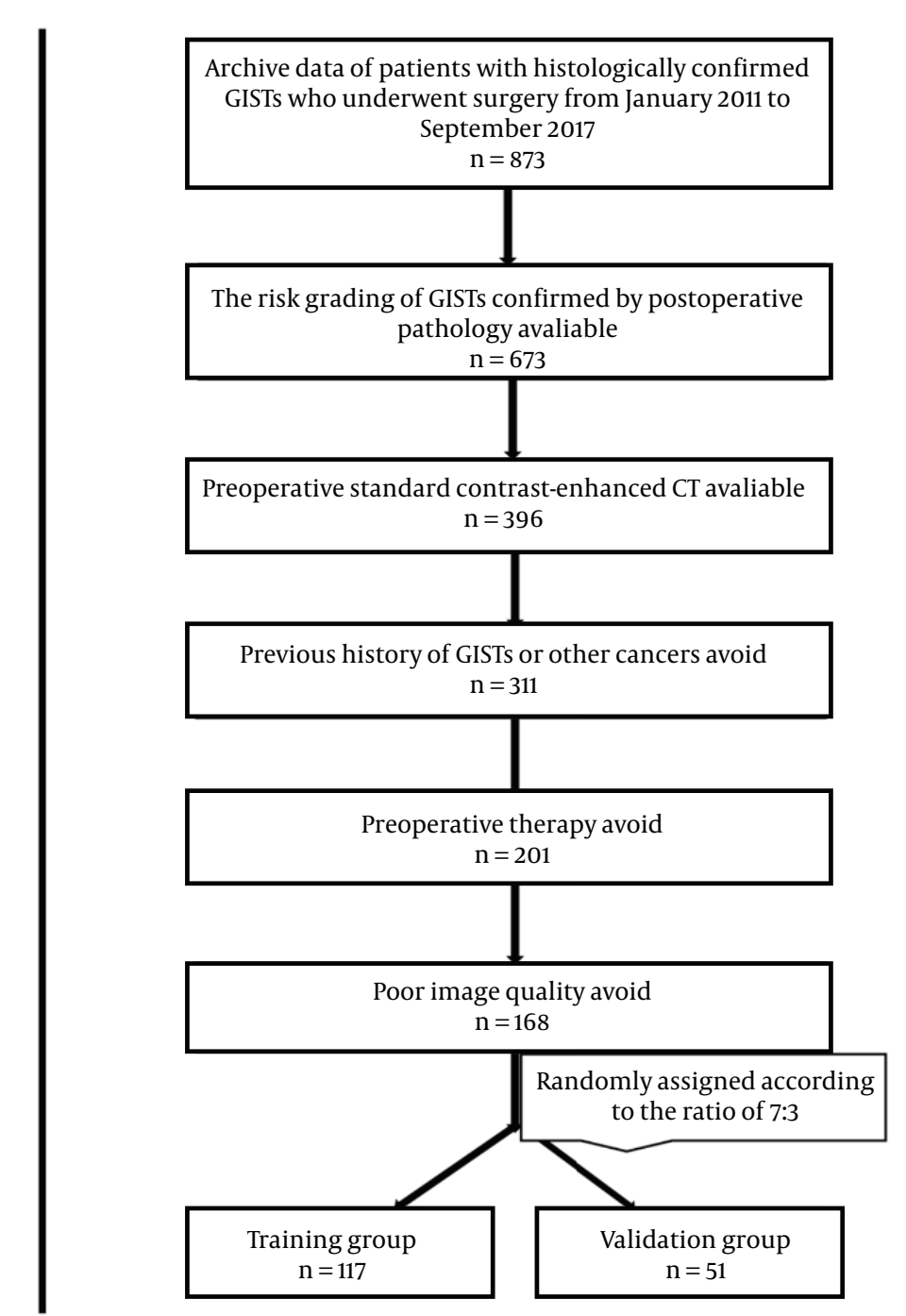

Archive data of patients with histologically confirmed September 2017 $\mathrm{n}=873$

Exclusion criteria:

1) previous history of GISTs or other

cancers;

2) preoperative

therap y(radiotherapy, chemotherapy or

chemoradiotherapy);

3) poor image quality affects lesion

segmentation.

Figure 1. Patient recruitment process

\subsection{Statistical Analysis}

Statistical analysis was performed in $\mathrm{R}$ (version 3.4.3, http://www. r-project. org) and SPSS 23.0 (IBM, Armonk, NY, USA). A two-tailed P value of $<0.05$ was used as the criterion to indicate a statistically significant difference. The Mann-Whitney U test was used to assess differences in patient age, whereas the $\chi^{2}$ test was used to assess differences in sex.

\subsection{Feature Selection and Prediction Model Establishment}

The radiomics signature and combination model were established in the training group. Univariate analysis was applied to the CT and texture features to identify the most relevant predictors of the grade of GISTs using Pearson's correlation test. Multivariate analysis was performed by least absolute shrinkage and selection operator (LASSO) regression to address multiple cross-related covariates and reduce the risk of overfitting of the data(18). The method of lambda selection was ten-fold cross-validation. The prediction models were developed by the linear fusion of selected features weighted by their coefficients, with a radiomics score (rad-score) and a prediction score (pre-score) calculated for each patient based on validation group.

\subsection{Prediction Performance}

The prediction performance of the models was evaluated by the area under the receiver operating characteristic (ROC) curve (AUC). The AUCs, sensitivity, specificity and 
Ren Cetal.

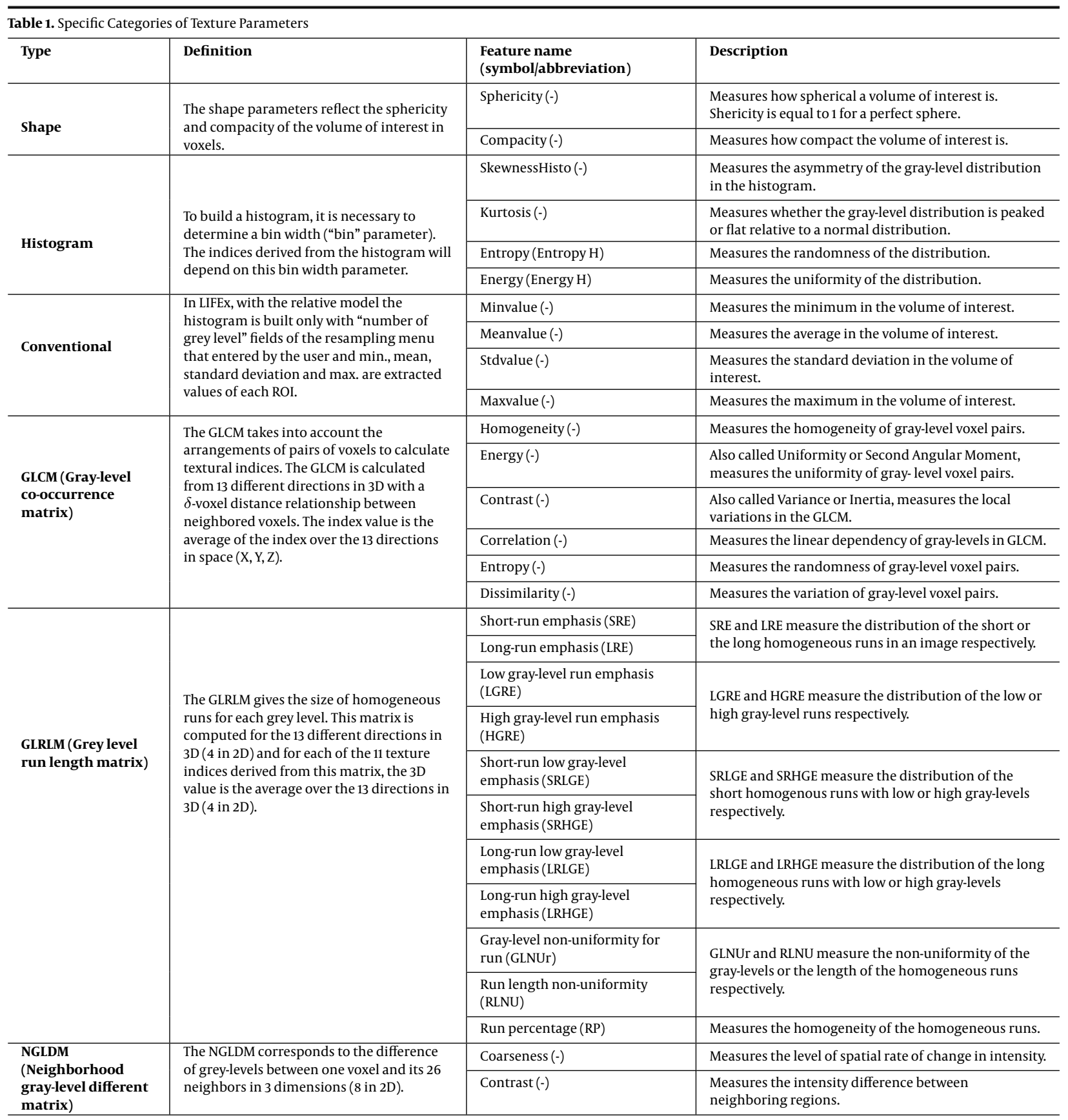

accuracy with $95 \%$ confidence intervals (CIs) were calculated for each model. Calibration was evaluated with the Hosmer-Lemeshow goodness-of-fit test, which reflects the consistency of the model forecast risk and the actual risk; $\mathrm{P}$ $>0.05$ indicated insignificant deviance from the theoretical perfect calibration. Intra- and interclass correlation coefficients (ICCs) were used to evaluate the consistency and reproducibility of the intra- and inter-observer agreement of the CT signs assessment and radiomics features extraction. An ICC greater than 0.75 indicated good consistency.

\section{Results}

\subsection{Patient Characteristics}

A total of 168 patients were enrolled in this study. The patient characteristics are summarized and compared in 

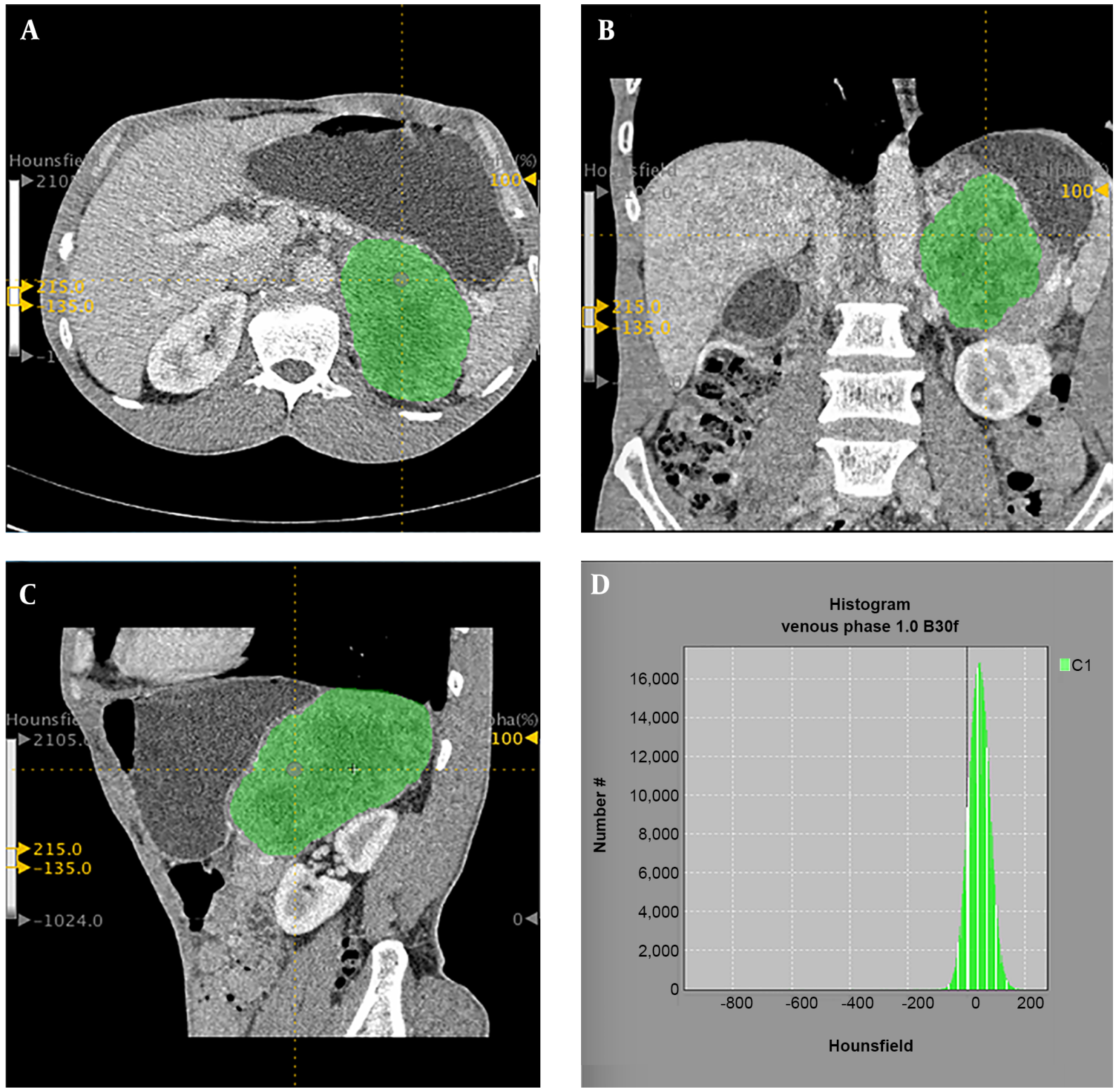

Figure 2. Abdominal portal venous-phase CT images of a 33-year-old man. Texture features were extracted from the primary tumor area (green overlay). A, Transverse section; B, Coronal section; C, Median sagittal section; D, Graylevel histogram.

Table 2. There were no significant differences in gender or age between the potential malignant group and the malignant group ( $P>0.05)$, consistent with the report (19).

\subsection{Establishment of the Radiomics Signature and Combina- tion Model}

A total of 29 texture features and seven CT parameters were extracted from the 168 patients' CT portal-phase images, and the agreement between the two radiologists was excellent for those features (all ICCs $>0.85)(\mathrm{P}<0.05)$. Thus, the mean measurement values of the two radiologists were used for further analysis. The cross-correlation matrixes (Figure 3A and B) showed that there were multiple complex cross-correlations among the 36 parameters. The selection of covariates with non-zero coefficients by Lasso regression to establish the two models was depicted in Figure $4 \mathrm{~A}$ and $\mathrm{B}$. The texture features of sphericity, compacity, contrast, dissimilarity and CT imaging features of 


\begin{tabular}{|c|c|c|c|}
\hline Variable & Potential malignant group $(n=66)$ & Malignant group $(n=102)$ & Pvalue \\
\hline Gender & & & $0.839^{\mathrm{a}}$ \\
\hline Female & 37 & 51 & \\
\hline Male & 29 & 51 & \\
\hline $\operatorname{Age}($ mean $\pm S D)$ & $58.8 \pm 9.61$ & $57.1 \pm 10.35$ & $0.701^{\mathrm{b}}$ \\
\hline
\end{tabular}

size, and location were selected to develop the predictive models. The rad- and pre-scores were calculated for each patient. Patients in the malignant group generally had higher rad-scores and pre-scores than patients in the potential malignant group (Table 3).

\subsection{Assessment and Comparison of the Prediction Models}

Both the radiomics signature and combination model were significantly associated with GIST grade. Regarding prediction performance, the combination model performed better than the radiomics signature (Table 4).

\subsection{Validation of the Prediction Models}

The Hosmer-Lemeshow goodness-of-fit test showed no statistically significant differences between the predictions and observations of the two models $(\mathrm{P}>0.05)$. The calibration curves of the two models in the validation group are shown in Figure 5.

\section{Discussion}

In this study, the value of conventional CT combined with texture analysis in grade of GISTs was discussed for the first time. A radiomic signature based on texture features and a combination model consisting of CT and texture parameters were established and used to identify predictive markers discriminating patients with potentially malignant and malignant GISTs. The combination model was shown to be more advantageous in this prediction.

The results of this study showed higher rad-scores and its compositions in patients with malignant GISTs. The sphericity is the maximum value 1 for a perfectly round ROI. When the ROI is another shape, the sphericity is less than 1 and not affected by regional translation, rotation and scale changes (17). The author considered this principle to explain the result that the sphericity was not significantly different between the two groups of patients. Contrast and dissimilarity are two parameters of GLCM.
Greater contrast and dissimilarity indicates a deeper texture groove, a larger image texture variation in different regions, and a more uneven local texture (20). Compared with the potential malignant GISTs, the compacity, contrast and dissimilarity of malignant GISTs were higher. We speculated that the results may be related to a higher degree of cell proliferation and higher blood supply compromise in the tumoral tissue. Those showed more mixed, compact and irregular texture associated with greater heterogeneity of the tumor region. Previous studies have confirmed that the texture features of human body could change due to pathological changes, and the above three parameters have been used to distinguish between benign or malignant lesions in patients with other tumors (21-23), suggesting that the texture of tumors with increased heterogeneity was more uneven.

This study also explored whether the prediction performance based on texture analysis could be improved by combination with conventional CT. The combination model was composed of the size and location of CT signs and the compacity of texture parameters, and this model was more advantageous than radiomics signature. The specificity and accuracy of the combination model were also superior. Tumor size and location have been confirmed in many reports to be closely related to the degree of malignancy $(24,25)$ : larger primary masses not in the stomach usually exhibit a higher degree of malignancy than smaller ones in the stomach. The results of this study confirm the hypothesis.

Liu et al. found that entropy based on venous-phase images can be used to distinguish between very low- and lowrisk GISTs (AUC = 0.684) (26). Nevertheless, no significant differences were detected in parameters other than those mentioned above between GISTs patients in this study. This discrepancy may be related to the selection of more dimensions and a wider range of texture parameters, leading to the parameters reduced and selected by LASSO being more representative. In addition, three-dimensional spatial analysis of the tumor ROI texture features could more 
A

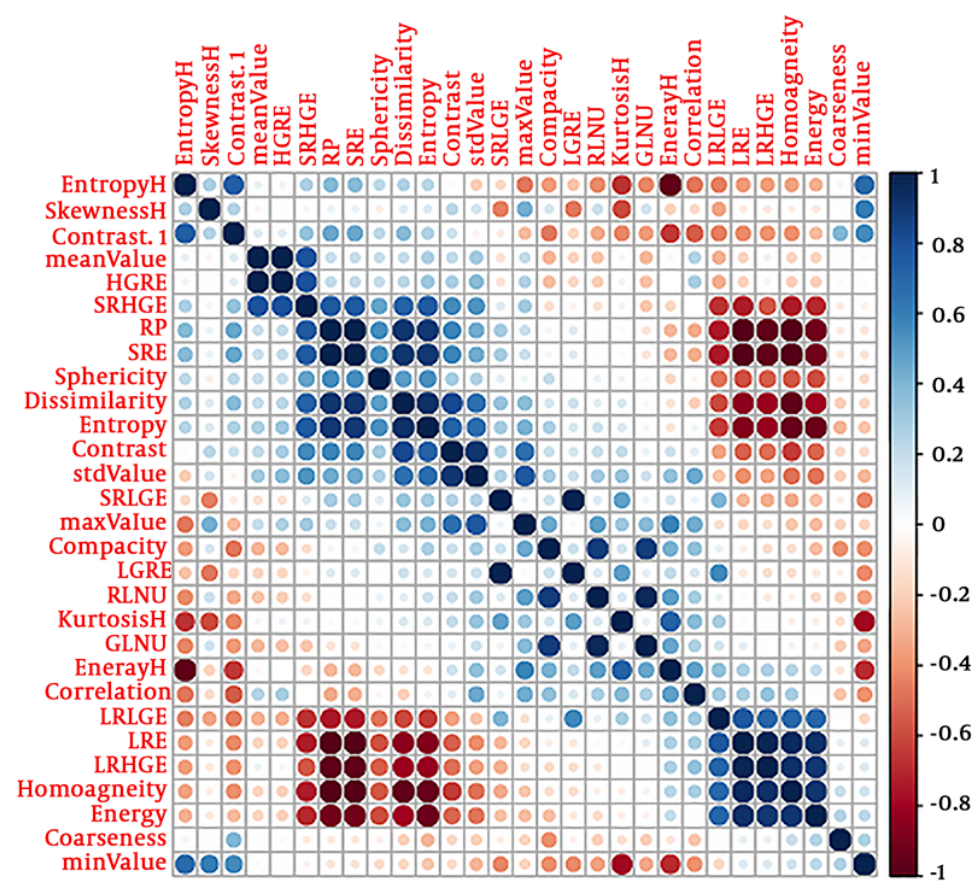

B

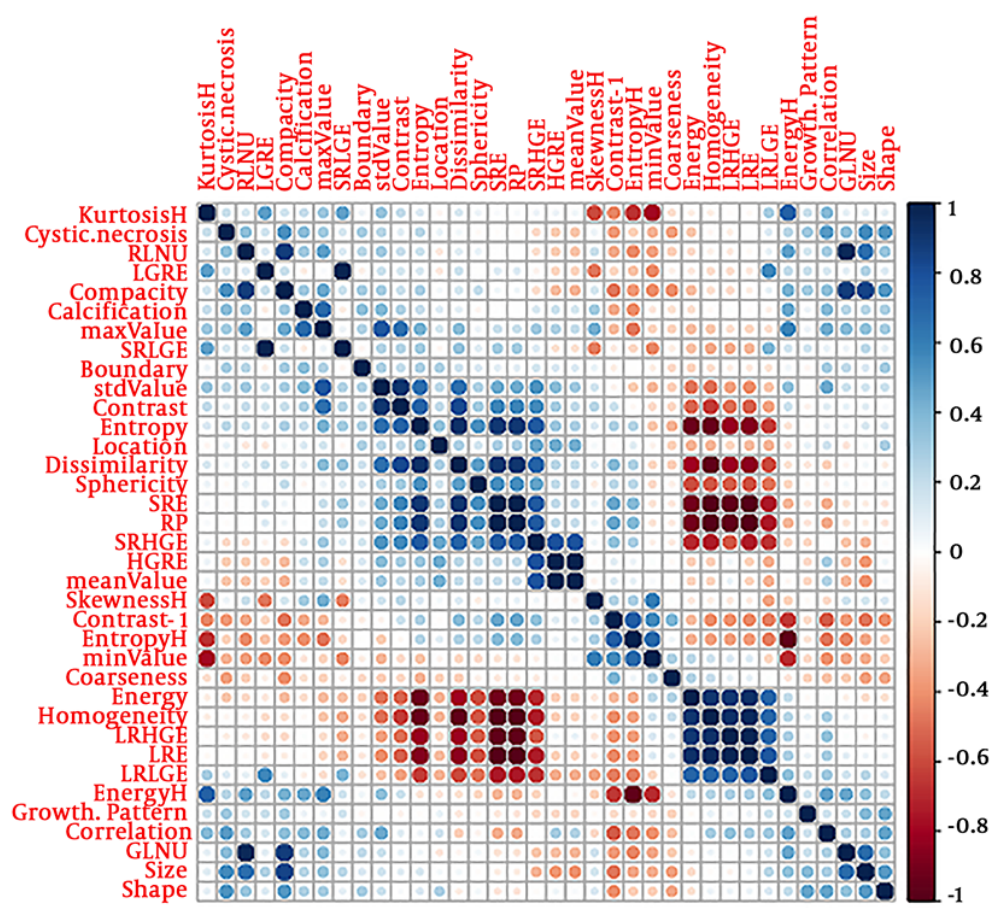

Figure 3. The cross-correlation matrix for covariates used to establish the radiomics signature (A) and combination model (B). The depth of color indicates the intensity of the correlation between covariates. The darker the color, the higher the correlation. The lighter the color, the lower the correlation. Blue represents positive correlation, and red represents negative correlation. 


\begin{tabular}{|c|c|c|c|c|}
\hline \multirow[t]{2}{*}{ Models } & \multicolumn{2}{|c|}{ Training group } & \multirow[t]{2}{*}{$Z$ value } & \multirow[t]{2}{*}{ Pvalue } \\
\hline & Potential malignant group $(n=45)$ & Malignant group $(n=72)$ & & \\
\hline Sphericity & $0.914(0.888,0.977)$ & $0.916(0.905,0.961)$ & -1.333 & 0.182 \\
\hline Compacity & $6.899(4.239,9.488)$ & $15.324(9.631,20.715)$ & -6.734 & $<0.001$ \\
\hline Contrast & $10.229(6.725,13.696)$ & $15.203(7.194,19.213)$ & -2.095 & 0.036 \\
\hline Dissimilarity & $2.362(1.947,2.853)$ & $2.729(2.729,3.415)$ & -2.280 & 0.023 \\
\hline Rad-score & $-1.606(-1.900,-0.682)$ & $0.388(-0.418,1.971)$ & -7.238 & $<0.001$ \\
\hline Pre-score & $-1.571(-1.577,-0.460)$ & $0.312(0.021,1.049)$ & -7.939 & $<0.001$ \\
\hline
\end{tabular}

${ }^{\mathrm{a}}$ Values are expressed as median (interquartile range [IQR]).

${ }^{\mathrm{b}} \mathrm{Z}$ and $\mathrm{P}$ values are the results of Mann-Whitney test.

A

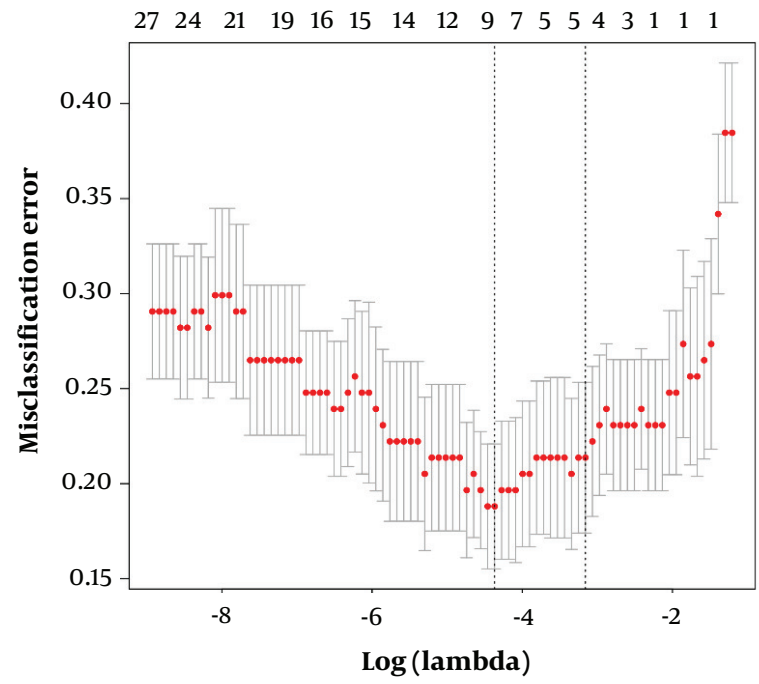

B

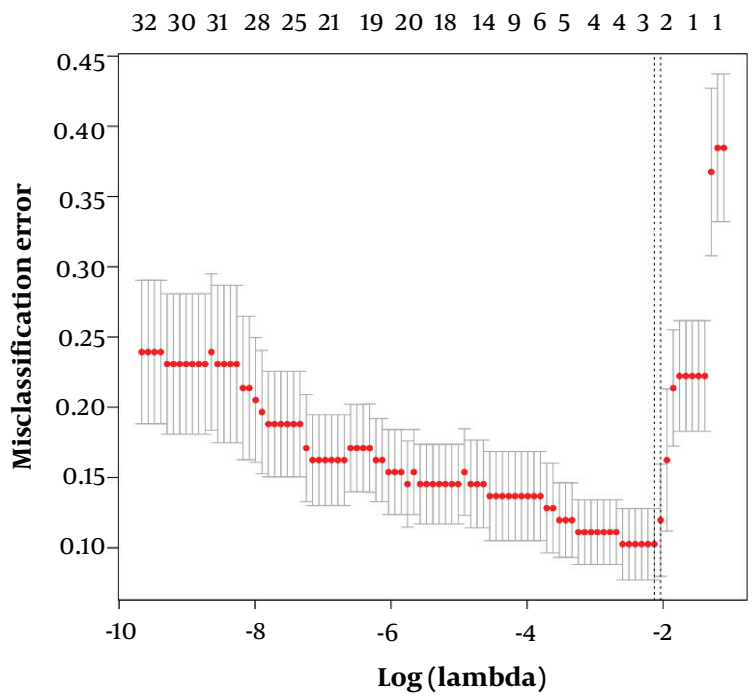

Figure 4. Feature selections for the radiomics signature (A) and combination model (B). Tuning parameter $(\lambda)$ selection in the lasso model used ten-fold cross-validation. The two vertical dashed lines represent one standard deviation on each side of the minimum value, corresponding to the chosen variables that better fit the models.

Table 4. Prediction Performance of the Radiomics Signature and Combination Model in the Validation Group ${ }^{\mathrm{a}}$

\begin{tabular}{|c|c|c|c|c|c|c|}
\hline Models & AUC, 95\% CI & Sensitivity, \% & Specificity, \% & Accuracy, \% & $Z$ value & Pvalue \\
\hline Radiomics signature & $0.897(0.811-0.983)$ & 76.20 & 90.00 & 84.30 & 1.85 & 0.06 \\
\hline Combination model & $0.959(0.905-1.000)$ & 90.50 & 93.30 & 90.20 & & \\
\hline
\end{tabular}

Abbreviations: AUC, area under the receiver operating characteristics curve; CI, confidence interval.

${ }^{\mathrm{a}} \mathrm{Z}$ and $\mathrm{P}$ values are the results of Mann-Whitney test.

accurately reflect the overall heterogeneity of the ROI (27).

The present study had several limitations. First, the sample size of this single-center retrospective study was relatively small, and the results need to be confirmed by further prospective and multicentric studies with larger sample sizes. Second, the features extracted in this study were only based on portal venous phase CT images.
Whether the use of other periods or some combination will increase the diagnostic efficiency requires further study.

In conclusion, the prediction models established in this study were validated to be valuable for grade evaluation in patients with GISTs and may represent non-invasive and practical biomarkers for optimizing the treatment strategy and improving the prognosis of GISTs. In addition, 


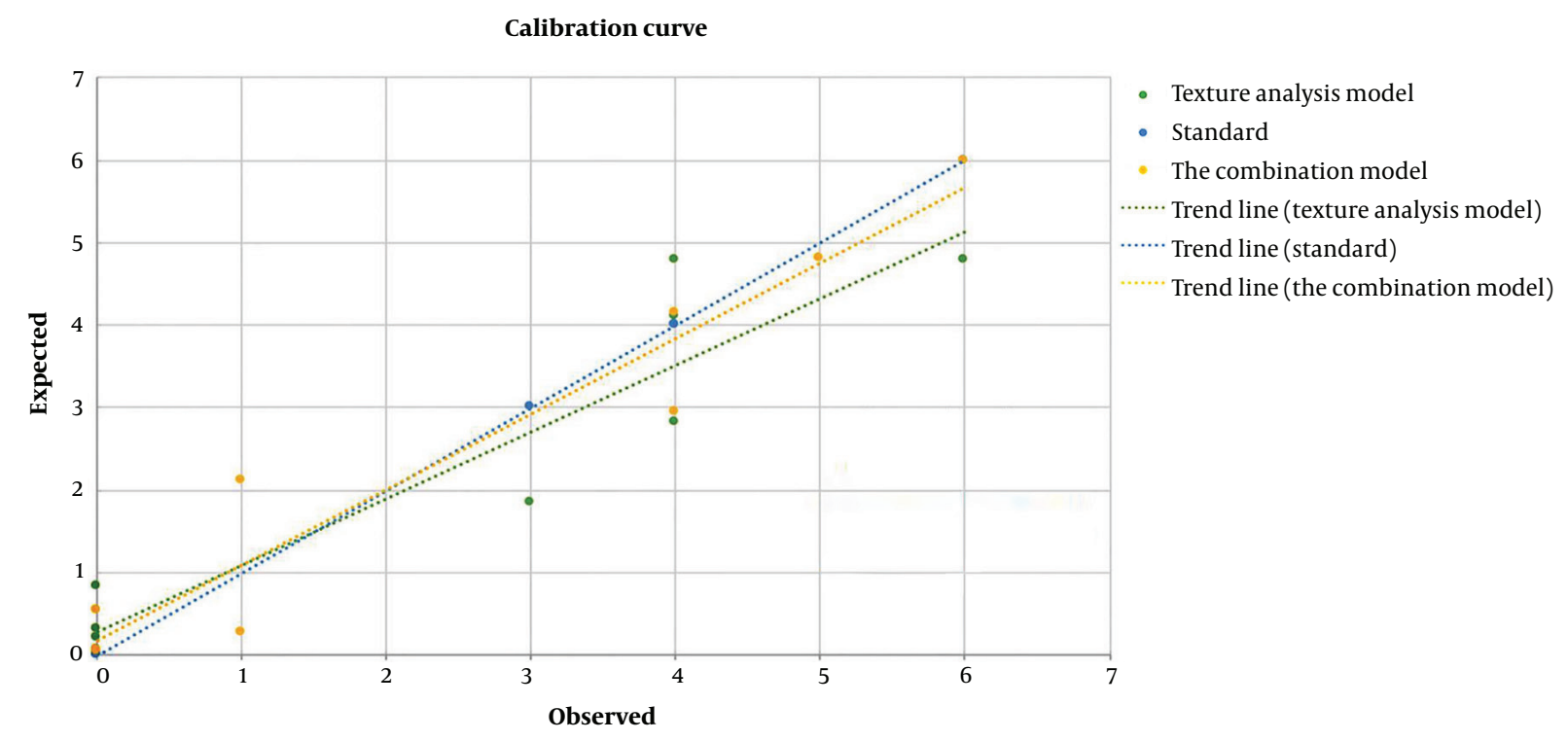

Figure 5. Calibration curves of the two models in the validation group. Calibration curves depict the calibration of the two prediction models in terms of agreement between the predicted probability of the gastrointestinal stromal tumor (GIST) grade and observed rate of grade.

the combination model had more advantages than texture analysis alone.

\section{Supplementary Material}

Supplementary material(s) is available here [To read supplementary materials, please refer to the journal website and open PDF/HTML].

\section{Acknowledgments}

All the authors have contributed significantly and have approved the manuscript.

\section{Footnotes}

Authors' Contributions: Shengjian Zhang developed the original idea and the protocol, abstracted and analyzed data, wrote the manuscript, and is guarantor. Caiyue Ren, Shengping Wang and Zhaoxia Jiang contributed to the development of the protocol, abstracted data, and prepared the manuscript.

Conflict of Interests: All the authors have no conflict of interest to disclose.

Ethical Approval: This study is a retrospective study and our institutional review board approved the ethical approval code.
Financial Disclosure: All the authors have no relevant financial interest to disclose.

Funding/Support: This work was supported by The National Natural Science Foundation of China (Grant Nos.: 81871347).

\section{References}

1. Flejou JF. [WHO Classification of digestive tumors: The fourth edition]. Ann Pathol. 2011;31(5 Suppl):S27-31. French. doi: 10.1016/j.annpat.2011.08.001. [PubMed: 22054452].

2. Joensuu H. Risk stratification of patients diagnosed with gastrointestinal stromal tumor. Hum Pathol. 2008;39(10):1411-9. doi: 10.1016/j.humpath.2008.06.025. [PubMed: 18774375].

3. Koo DH, Ryu MH, Kim KM, Yang HK, Sawaki A, Hirota S, et al. Asian consensus guidelines for the diagnosis and management of gastrointestinal stromal tumor. Cancer Res Treat. 2016;48(4):115566. doi: 10.4143/crt.2016.187. [PubMed: 27384163]. [PubMed Central: PMC5080813].

4. Demetri GD, von Mehren M, Antonescu CR, DeMatteo RP, Ganjoo KN, Maki RG, et al. NCCN task force report: Update on the management of patients with gastrointestinal stromal tumors. J Natl Compr Canc Netw. 2010;8 Suppl 2:S1-41. quiz S42-4. doi: 10.6004/jnccn.2010.0116. [PubMed: 20457867]. [PubMed Central: PMC4103754].

5. Tokumoto N, Tanabe K, Misumi T, Fujikuni N, Suzuki T, Ohdan H. The usefulness of preoperative 18FDG positron-emission tomography and computed tomography for predicting the malignant potential of gastrointestinal stromal tumors. Dig Surg. 2014;31(2):79-86. doi: 10.1159/000357149. [PubMed: 24776690].

6. Kang TW, Kim SH, Jang KM, Choi D, Ha SY, Kim KM, et al. Gastrointestinal stromal tumours: Correlation of modified NIH risk stratification with diffusion-weighted MR imaging as an imaging biomarker. Eur 
J Radiol. 2015;84(1):33-40. doi: 10.1016/j.ejrad.2014.10.020. [PubMed: 25466773].

7. Vasconcelos RN, Dolan SG, Barlow JM, Wells ML, Sheedy SP, Fidler JL, et al. Impact of CT enterography on the diagnosis of small bowel gastrointestinal stromal tumors. Abdom Radiol (NY). 2017;42(5):1365-73. doi: 10.1007/s00261-016-1033-z. [PubMed: 28058449].

8. Lambin P, Rios-Velazquez E, Leijenaar R, Carvalho S, van Stiphout RG, Granton P, et al. Radiomics: Extracting more information from medical images using advanced feature analysis. Eur J Cancer. 2012;48(4):441-6. doi: 10.1016/j.ejca.2011.11.036. [PubMed: 22257792]. [PubMed Central: PMC4533986].

9. Hodgdon T, McInnes MD, Schieda N, Flood TA, Lamb L, Thornhill RE. Can quantitative CT texture analysis be used to differentiate fat-poor renal angiomyolipoma from renal cell carcinoma on unenhanced CT images? Radiology. 2015;276(3):787-96. doi: 10.1148/radiol.2015142215. [PubMed: 25906183].

10. Fujita A, Buch K, Li B, Kawashima Y, Qureshi MM, Sakai O. Difference between HPV-Positive and HPV-negative non-oropharyngeal head and neck cancer: Texture analysis features on CT. J Comput Assist Tomogr. 2016;40(1):43-7. doi: 10.1097/RCT.0000000000000320. [PubMed: 26466116].

11. Liu L, Liu Y, Xu L, Li Z, Lv H, Dong N, et al. Application of texture analysis based on apparent diffusion coefficient maps in discriminating different stages of rectal cancer. J Magn Reson Imaging. 2017;45(6):1798808. doi: 10.1002/jmri.25460. [PubMed: 27654307].

12. Ba-Ssalamah A, Muin D, Schernthaner R, Kulinna-Cosentini C, Bastati N, Stift J, et al. Texture-based classification of different gastric tumors at contrast-enhanced CT. Eur J Radiol. 2013;82(10):e537-43. doi: 10.1016/j.ejrad.2013.06.024. [PubMed: 23910996].

13. Ma Z, Fang M, Huang Y, He L, Chen X, Liang C, et al. CT-based radiomics signature for differentiating Borrmann type IV gastric cancer from primary gastric lymphoma. Eur J Radiol. 2017;91:142-7. doi: 10.1016/j.ejrad.2017.04.007. [PubMed: 28629560].

14. Zhou Y, He L, Huang Y, Chen S, Wu P, Ye W, et al. CT-based radiomics signature: a potential biomarker for preoperative prediction of early recurrence in hepatocellular carcinoma. Abdom Radiol (NY). 2017;42(6):1695-704. doi: 10.1007/s00261-017-1072-0. [PubMed: 28180924].

15. Ahn SJ, Kim JH, Park SJ, Han JK. Prediction of the therapeutic response after FOLFOX and FOLFIRI treatment for patients with liver metastasis from colorectal cancer using computerized CT texture analysis. Eur J Radiol. 2016;85(10):1867-74. doi: 10.1016/j.ejrad.2016.08.014. [PubMed: 27666629].

16. Takao H, Yamahira K, Doi I, Watanabe T. Gastrointestinal stromal tumor of the retroperitoneum: CT and MR findings. Eur Radiol. 2004;14(10):1926-9. doi: 10.1007/s00330-004-2404-3. [PubMed: 15300393].
17. Orlhac F, Nioche C, Soussan M, Buvat I. Understanding changes in tumor texture indices in PET: A comparison between visual assessment and index values in simulated and patient data. $J$ Nucl Med. 2017;58(3):387-92. doi: 10.2967/jnumed.116.181859. [PubMed: 27754906].

18. Tibshirani R. Regression shrinkage and selection Via the Lasso. J R Stat Soc Series B Methodol. 1996;58(1):267-88. doi: 10.1111/j.25176161.1996.tb02080.x.

19. Sandrasegaran K, Rajesh A, Rydberg J, Rushing DA, Akisik FM, Henley JD. Gastrointestinal stromal tumors: Clinical, radiologic, and pathologic features. AJR Am J Roentgenol. 2005;184(3):803-11. doi: 10.2214/ajr.184.3.01840803. [PubMed: 15728601].

20. Haralick RM, Shanmugam K, Dinstein I. Textural features for image classification. IEEE Trans Cybern. 1973;SMC-3(6):610-21. doi: 10.1109/tsmc.1973.4309314.

21. Alvarenga AV, Pereira WC, Infantosi AF, Azevedo CM. Complexity curve and grey level co-occurrence matrix in the texture evaluation of breast tumor on ultrasound images. Med Phys. 2007;34(2):379-87. doi: 10.1118/1.2401039. [PubMed: 17388154].

22. Cozzi L, Dinapoli N, Fogliata A, Hsu WC, Reggiori G, Lobefalo F, et al Radiomics based analysis to predict local control and survival in hepatocellular carcinoma patients treated with volumetric modulated arc therapy. BMC Cancer. 2017;17(1):829. doi: 10.1186/s12885-017-3847-7. [PubMed: 29207975]. [PubMed Central: PMC5718116].

23. Eilaghi A, Baig S, Zhang Y, Zhang J, Karanicolas P, Gallinger S, et al $\mathrm{CT}$ texture features are associated with overall survival in pancreatic ductal adenocarcinoma - a quantitative analysis. BMC Med Imaging. 2017;17(1):38. doi: 10.1186/s12880-017-0209-5. [PubMed: 28629416]. [PubMed Central: PMC5477257].

24. Miettinen M, Sobin LH, Lasota J. Gastrointestinal stromal tumors of the stomach: A clinicopathologic, immunohistochemical, and molecular genetic study of 1765 cases with long-term follow-up. Am J Surg Pathol. 2005;29(1):52-68. doi: 10.1097/01.pas.0000146010.92933.de. [PubMed: 15613856].

25. Gronchi A. Risk stratification models and mutational analysis: Keys to optimising adjuvant therapy in patients with gastrointestinal stromal tumour. Eur J Cancer. 2013;49(4):884-92. doi: 10.1016/j.ejca.2012.10.025. [PubMed: 23206668].

26. Liu S, Pan X, Liu R, Zheng H, Chen L, Guan W, et al. Texture analysis of CT images in predicting malignancy risk of gastrointestinal stromal tumours. Clin Radiol. 2018;73(3):266-74. doi: 10.1016/j.crad.2017.09.003. [PubMed: 28969853].

27. Ng F, Ganeshan B, Kozarski R, Miles KA, Goh V. Assessment of primary colorectal cancer heterogeneity by using whole-tumor texture analysis: Contrast-enhanced CT texture as a biomarker of 5-year survival. Radiology. 2013;266(1):177-84. doi: 10.1148/radiol.12120254. [PubMed: 23151829]. 